\title{
Influência do efeito antropogênico nos padrões dominantes de atuação dos ciclones extratropicais no Hemisfério Sul
}

\section{Influence of the anthropogenic effect on the dominant patterns of extratropical cyclones activity in the Southern Hemisphere}

\author{
Rose Ane Pereira de Freitas ${ }^{1 *}$, Fernanda Casagrande ${ }^{2}$, Douglas da Silva Lindemann ${ }^{1}$, Jéferson
} Prietsch Machado ${ }^{3}$, Jackson Martins Rodrigues ${ }^{4}$,Flávio Barbosa Justino ${ }^{5}$

\begin{abstract}
RESUMO
Nesse estudo utiliza-se simulações numéricas do modelo ECHAM5/MPI-OM para identificar os padrões dominantes de energia cinética com o objetivo de explicar a gênese e manutenção das trajetórias preferenciais dos ciclones extratropicais (Storm Tracks, ST) no Hemisfério Sul, localizando suas origens e apresentando suas trajetórias preferenciais através do vetor $\vec{E}$. Os resultados mostraram que para um cenário futuro, os ST parecem estar ligados diretamente ao fluxo de oeste, podendo-se afirmar que em um cenário futuro haverá uma intensificação desse transporte e os mesmos poderão deslocar-se mais para Sul. Dessa forma, espera-se que haja implicações nos abastecimentos de água de algumas regiões como o Sul da África, onde maior parte da população é dependente da precipitação na região. No Sul do Brasil, os resultados indicam aumentos nas chuvas durante os meses de verão, contrastando com uma diminuição na frequência dos ST e aumento na intensidade no inverno. Sugerimos que essas mudanças podem impactar a dinâmica climática do litoral sul brasileiro, com magnitude ainda desconhecida.
\end{abstract}

Palavras-chave: Storm Tracks; Fluxo de oeste; Energia cinética; Mudanças Climáticas.

\section{ABSTRACT}

In this study, we used ECHAM5/MPI-OM numerical simulations to identify the dominant patterns of kinetic energy in order to explain the genesis and maintenance of the preferential trajectories of the extratropical cyclones (Storm Tracks, ST) in the Southern Hemisphere. Furthermore, we present the preferred trajectories, origins and localization through the vector E. The results for future scenario showed that ST is widely related to the western flow. Thus, considering the future scenarios, it is expected both intensification and displacement of this transport toward south. In this way, we suggest for future important implications for the water supplies of some regions such as southern Africa, where most of the population is dependent on rainfall. In southern Brazil, the results indicate increases in rainfall during the summer months, contrasting with a decrease in ST frequency and an increase in intensity in wintertime. Additionally,

\footnotetext{
${ }^{1}$ Faculdade de Meteorologia, Universidade Federal de Pelotas, Pelotas-RS, Brasil. pfreitas.rose@gmail.com

${ }^{2}$ Divisão de Modelagem Numérica do Sistema Terrestre - Instituto Nacional de Pesquisas Espaciais (INPE), Instituto Nacional de Pesquisas Espaciais, Cachoeira Paulista, Brasil ${ }^{3}$ Instituto de Oceanografia / Programa de Pós-Graduação em Oceanologia, Universidade Federal do Rio Grande (FURG) - Rio Grande - Brasil

${ }^{4}$ Departamento de Geografia e Políticas Públicas, Universidade Federal Fluminense, Angra dos Reis-RJ, Brasil.

${ }^{5}$ Universidade Federal de Viçosa - Departamento de Engenharia Agrícola - Viçosa - Brasil
} 
we suggest that these changes may impact the climate dynamics of the southern Brazilian coast, with unknown magnitude.

Keywords: Storm Tracks; Westerlies; Kinetic energy; Climate change.

\section{INTRODUÇÃO}

As regiões de latitudes médias e altas são influenciadas diretamente pelos ciclones extratropicais por possuírem a capacidade de alterar a condição de tempo e clima de determinada região. As faixas predominantes de atuação dos ciclones extratropicais, conhecidas como "Storm Tracks" (ST) desempenham um papel dominante na circulação atmosférica global. Sendo responsáveis pela redistribuição de calor entre equador e os polos, os ST exercem forte influência na radiação incidente, precipitação e cobertura de nuvens através dos processos de troca de calor, momentum e vapor d'água (Freitas et al., 2019; LAU, 1988; TING; HELD, 1990; Machado et al., 2021).

Importantes relatórios internacionais e um expressivo número de publicações científicas têm alertado sobre o impacto das mudanças climáticas e o provável aumento de eventos climáticos extremos (IPCC -AR6; McBean 2004; Roberts et al. 2020). De acordo com O’Gorman et al., (2010), as mudanças nos padrões dominantes de atuação dos ST simuladas para o clima futuro, devido ao efeito antropogênico tem potencial de afetar a circulação atmosférica com impactos diretos nas atividades humanas e significativos impactos socioeconômicos. Esse é considerado um hot topic no âmbito de mudanças climáticas e carece de respostas conclusivas.

Dentre os diversos avanços científicos que aconteceram nas últimas décadas, a evolução dos modelos climáticos e do sistema terrestre tem destaque. As simulações numéricas dos modelos climáticos são importantes ferramentas para investigação do clima passado, presente e futuro. Além disso, permitem análises da resposta do clima global ao efeito antropogênico, como por exemplo, aumento da concentração dos gases do efeito estufa. De acordo com Lehmann et al. (2014), às mudanças climáticas projetadas tem grande potencial de afetar os padrões dominantes de atuação dos ST associados a mudanças no transporte de energia, momentum de vapor d'água.

Freitas et al (2019), mostraram através de um experimento numérico para o cenário futuro considerando uma concentração de $\mathrm{CO} 2$ atmosférico de aproximadamente 
770 ppm (partes por milhão) um deslocamento de Storm Tracks (ST) de $5^{\circ}$ latitude em direção ao sul e mudanças do transporte meridional de calor sensível, próximo a $50{ }^{\circ} \mathrm{S}$ induzidos pelo aumento da SST intensificando a região de ST e consequentemente gerando aumento na ocorrência de ciclones extratropicais. De maneira geral, os resultados indicam mudança no padrão de ocorrência da atividade ciclogenética, com eventos menos frequentes, porém mais intensos.

Padrões de teleconexões atmosféricas, como o fenômeno El Niño-Oscilação Sul (ENSO), podem afetar a instabilidade baroclínica na região de ST. Machado et al (2021) mostraram que os ST apresentam variabilidade sazonal durante os eventos de ENOS, resultando em ciclones extratropicais mais intensos no Hemisfério Sul com trajetórias em direção a latitudes mais baixas (mais altas) durante os verões do El Niño (La Niña), por outro lado pouca diferença foi notada nos invernos sob atuação de El Niño e La Niña.

Logo, a dinâmica dos ST é associada a regiões de geração baroclínica, que se formam devido à conversão de energia potencial do estado básico para energia cinética (EKE). Nesse trabalho utiliza-se simulações numéricas do modelo ECHAM5/MPI-OM para identificar os padrões dominantes da EKE e localizar aorigem de tais perturbações, mostrando seus caminhos preferenciais através do vetor $\vec{E}$.

\section{MATERIAIS E MÉTODOS}

A análise do clima atual foi realizada através de estatísticas construídas com base em reanálises obtidas junto ao "European Centre For Medium-Range Weather Forecast" (ECMWF). O centro disponibiliza produtos de reanálises constituindo o ERA-40, que é um produto resultante da reavaliação das condições para a atmosfera e a superfície, compreendendo um período de janeiro de 1957 até o período atual. A reanálise do ERA40 possui uma resolução vertical de 60 níveis e uma resolução horizontal de aproximadamente $110 \mathrm{~km}$ (T159), resolução temporal de 6 horas e espaçamento de grade de $1,125^{\circ}$ x $1,125^{\circ}$ (Uppala, 2005). Neste estudo o período analisado foi de 1980 a 2000.

As simulações numéricas utilizadas neste trabalho foram realizadas pelo modelo acoplado ECHAM5/MPI-OM, para período atual (PD) e sob condições de aquecimento global (GHG). O cenário futuro (GHG) inclui concentração de Dióxido de Carbono atmosférico $(\mathrm{CO} 2)$ de aproximadamente 700 ppm, relativos aos níveis pré-industriais de aproximadamente 370 ppm. Estas simulações são parte do projeto ESSENCE (Sterl et al. 2008, www.knmi.nl/^sterl/Essence). O ECHAM5/MPI-OM é um modelo acoplado que 
possui uma componente atmosférica - ECHAM5 e outra oceânica - MPI-OM. O modelo é descrito em detalhes em Jungclaus et al., (2006).

Modelos climáticos podem ser utilizados para fins de previsão e também para estudos de processos climáticos. A habilidade do modelo ECHAM5/MPI-OM em reproduzir sazonalmente o clima do HS no presente foi previamente avaliada aqui e posteriormente utilizada nas projeções do clima futuro, com incremento de mais de 100 anos de simulação que inclui o efeito androgênico através do aumento de CO2. Para este estudo foram utilizados dados entre os anos de 1981 e 2000 considerados como presente (PD) e simulações para um cenário futuro para os anos entre 2080 e 2100 (GHG).

A região deste estudo localiza-se no Hemisfério Sul abrangendo desde a região extratropical até a região polar, entre as latitudes $40{ }^{\circ} \mathrm{S}$ e $90{ }^{\circ} \mathrm{S}$. A partir de dados de reanálise ERA-40, diversas variáveis (não mostradas aqui) foram previamente comparadas com os dados do modelo, e mostraram uma boa acurácia concordando com Bengtsson et al. (2006). Essa comparação entre modelo e reanálise é usada para fins validação dos dados é apresentada para dados de temperatura do ar no nível de $500 \mathrm{hPa}$ na Figura 1, juntamente com a região de estudo.

Para as análises da variabilidade e dos modos dominantes dos campos simulados, sua variação no tempo, e a quantificação da importância relativa desses padrões por meio da variância explicada, utilizamos o método das Funções Ortogonais Empíricas (EOFEmpirical Orthogonal Functions), também conhecido de Análise de Componentes Principais (ACP) (Kutzbach, 1967). A técnica de Funções Ortogonais Empíricas (EOFs) tem sido usada para analisar diversos campos escalares e para se estudar os padrões atmosféricos dominantes relacionados com fenômenos de várias escalas de tempo e espaço (LEGLER, 1983).

Segundo Mesquita (2006) os ST estão associados a regiões de geração baroclínica que se formam devido à conversão de energia potencial do estado básico para energia cinética. Isto ocorre por conta do movimento do ar quente ascendente proveniente dos trópicos, na direção do polo e do ar frio descendente na direção do equador conduzido em ondas baroclínicas e sistemas frontais. Considerando que quatro tipos de energia são importantes para determinar o transporte meridional de energia, a energia interna é a energia associada com a temperatura da atmosfera e a energia potencial associada com o potencial gravitacional do ar, em alguma distância, acima da superfície. 
Figura 1 - Região de estudo sobre dados de Temperatura média do ar $\left({ }^{\circ} \mathrm{C}\right) 500 \mathrm{hPa}$. (a) Dados de reanálise ERA_40 em DJF; (b) dados simulados pelo modelo ECHAM5/MPI-ON para o presente em DJF; e (c) e (d) o mesmo para os meses de inverno.

(a) $\mathrm{t}$-DJF-ERA-40

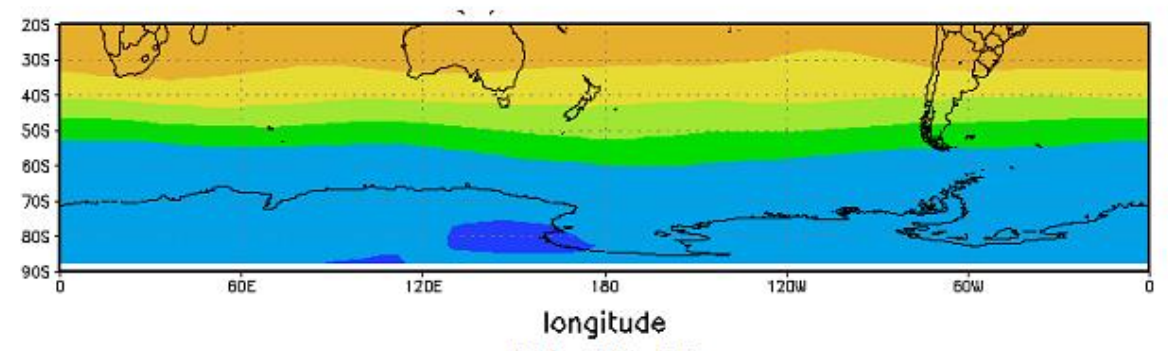

(a)

(b) $t$-DJF - PD

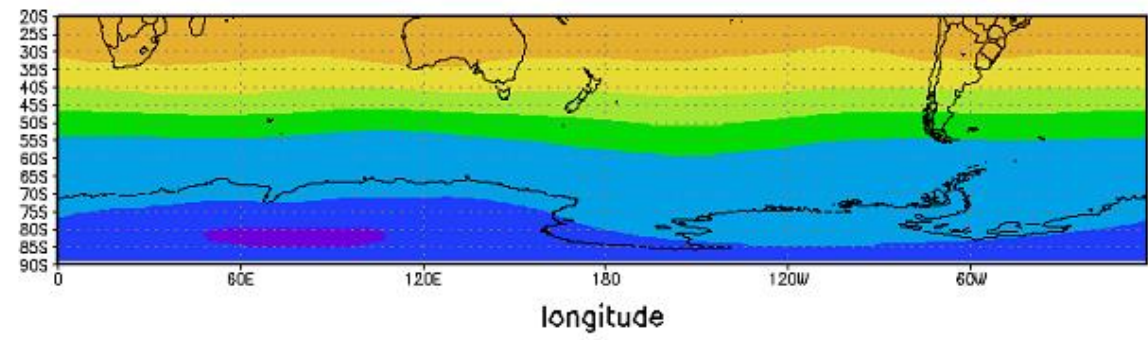

(b)

(c) $t$ - JJA - ERA-40

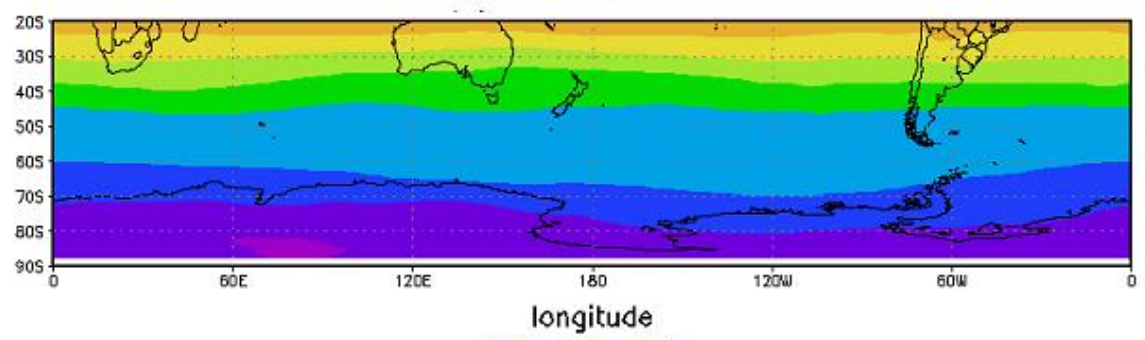

(c)

(d) $t-J J A-P D$

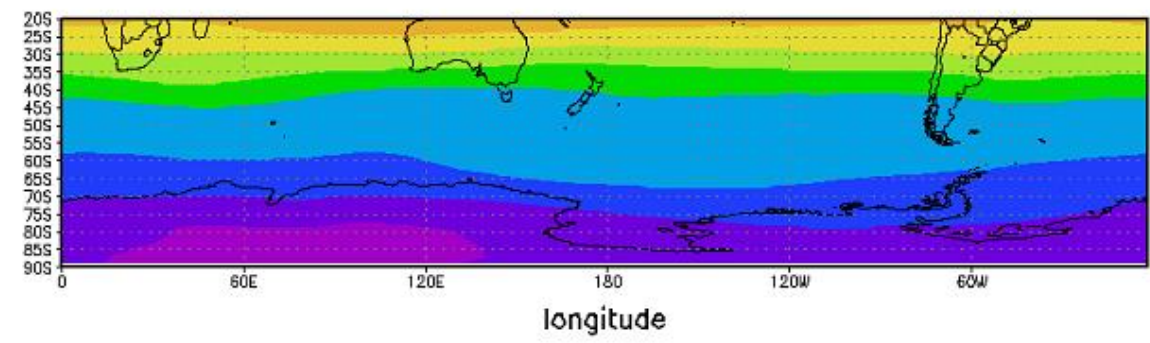

(d)

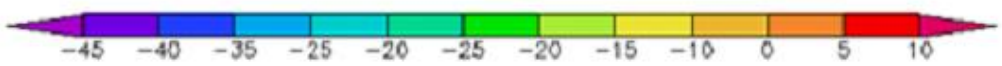

Fonte: Dos autores. 
A associação da energia interna e a potencial constituem em torno de $97 \%$ da energia da atmosfera. Embora a energia cinética represente uma pequena fração da energia total, ela ainda é muito importante para entender a gênese e manutenção dos ST, porque os movimentos são os meios pelo qual a energia é transportada do equador para os polos (HARTMAN, 1994). O estudo da energia cinética por unidade de massa (EKE), ao longo de uma determinada latitude, é obtido segundo o método definido por Haltiner e Williams (1980), conforme mostrado na Equação 1 (E1).

$$
\left[E K E=\frac{1}{2}\left(\overline{\left.u^{\prime 2}+v^{\prime 2}\right)}\right]\right.
$$

Onde, o primeiro termo da equação corresponde à energia cinética do escoamento zonal (2/2); o segundo termo, energia cinética do escoamento meridional (2/2); e a barra, média temporal.

Os turbilhões têm influência direta sobre os transportes de calor e momentum, e essas influências geralmente podem ser resumidas pelo vetor $\vec{E}$. Logo, este é utilizado para analisar as influências dos vórtices dos ST no fluxo de oeste. Esta avaliação do vetor $\vec{E}$, inicialmente proposta por Hoskins e Valdes (1990), quantifica a forma de propagação, e explica a interação do fluxo de vórtices transientes. Além disso, ele também mede a propagação de vórtices alongados meridionalmente em relação ao fluxo de oeste médio. Nas regiões onde $\vec{E}$ é divergente (convergente), há uma força horizontal da circulação média de acordo com uma tendência dos turbilhões a aumentar (diminuir) a oeste do Fluxo Médio (HALL et al., 1994).

O transporte horizontal de momentum representa a maior parte da componente meridional do vetor $\vec{E}$, enquanto que os transportes meridionais de calor constituem a parte vertical do vetor $\vec{E}$, sendo um dos principais fluxos associados com instabilidade baroclínica. Além disso, o vetor $\vec{E}$ tem como objetivo localizar a origem de tais perturbações, mostrando seus caminhos preferenciais. Logo, para analisar a influência dos ST sobre o fluxo de Oeste usaremos:

$$
\left[E=\left(\overline{v^{\prime 2}-u^{\prime 2}}, \overline{-u^{\prime} v^{\prime}}\right)\right]
$$

Onde, u'v' mostra os transportes de momentum pelos turbilhões e representa a maior parte da componente meridional do fluxo de E. Mudanças nos ST também significam mudanças no fluxo de calor transiente de um turbilhão em direção aos polos e consequentemente no vetor $\vec{E}$. Há uma influência direta dos turbilhões através do 
transporte de calor e momentum que geralmente é resumida por este vetor $\vec{E}$, que então será usado neste estudo para analisar a influência dos vórtices dos ST nos ventos de oeste.

Os campos de anomalias utilizados foram obtidos pela diferença entre as simulações dos cenários futuros e passado. Em estudos climáticos, o conceito de anomalia é frequentemente empregado, em virtude do interesse das pesquisas em investigar o quanto o clima de determinada região difere do que é considerado normal (IPCC-AR6; PEZZI; SOUZA, 2005). Deste modo, a anomalia de uma determinada variável pode ser definida como a diferença (ou desvio) entre a variável observada para um determinado local, durante certo período e um valor médio, previamente calculado com dados de um longo período, para esse mesmo local.

\section{RESULTADOS E DISCUSSÕES}

A Figura 2 mostra os padrões dominantes da EKE determinada através do cálculo da EOF para os meses de verão e inverno para as simulações PD e GHG para os meses de inverno e verão. As simulações para o verão PD e GHG são apresentadas na Figura 2 (a) e (b). Robustos padrões de variações foram identificadas entre as latitudes de $30{ }^{\circ} \mathrm{S}$ e $65^{\circ} \mathrm{S}$, e longitudes de $90^{\circ} \mathrm{E}$ e $120^{\circ} \mathrm{E}$, com valores máximos de amplitude entre 20 e 22 $\mathrm{m}^{2} \mathrm{~s}^{-2}$.. As simulações GHG (Figura $2 b$ ) demonstram que o padrão dominante apresentase entre as mesmas latitudes porém deslocados mais ao sul da Austrália onde seus máximos de EKE aproximam-se de $27 \mathrm{~m}^{2} \mathrm{~s}^{-2}$.

Para os meses de inverno nas simulações PD (Figura 2c), nota-se que as maiores variações de EKE apresentam-se sobre os Oceanos Atlântico e Índico com valores máximos próximos a $22 \mathrm{~m}^{2} \mathrm{~s}^{-2}$, demonstrando um acréscimo nas variações em relação aos meses de verão, sendo mais intensas a leste do Oceano Atlântico e Oceano Índico. Para as simulações GHG o padrão dominante se estende por uma faixa maior de latitudes entre $35{ }^{\circ} \mathrm{S}$ e $55{ }^{\circ} \mathrm{S}$ evidenciando-se sobre o sul do Oceano Índico, sul da Austrália, e com máximos destacados sobre o Oceano Atlântico.

Os padrões dominantes de EKE mostram que, para o cenário futuro analisado, as maiores tempestades estariam distribuídas no verão a partir de $60{ }^{\circ} \mathrm{E}$ até $120{ }^{\circ} \mathrm{W}$, sugerindo maior impacto sobre o sul da Austrália e Nova Zelândia. Nos meses de inverno as maiores tempestades apresentam-se entre o sul da África, Oceano Índico e sul da Austrália e bem mais evidentes no Oceano Atlântico. 
Essa tendência a maiores tempestades no Oceano Atlântico corroboram com resultados apresentados em estudos anteriores, como Pezza e Siammonds (2005), que atribuíram à Oscilação Antártica no seu modo/índice positivo, acima dos valores médios um aquecimento do litoral sul brasileiro, reforçando a convecção. Em um cenário de aquecimento global o aumento da Temperatura da Superfície do pode favorecer a intensificação dos ST, aumentando a probabilidade de ocorrência dos ciclones extratropicais no Atlântico Sul.

Figura 2. Padrão espacial da EKE a partir do cálculo de EOF (a) Dados de reanálise ERA-40 em DFJ, (b) Dados simulados pelo modelo ECHAM5/MPI-ON para GHG em DJF e (c, d) o mesmo para os meses de inverno.

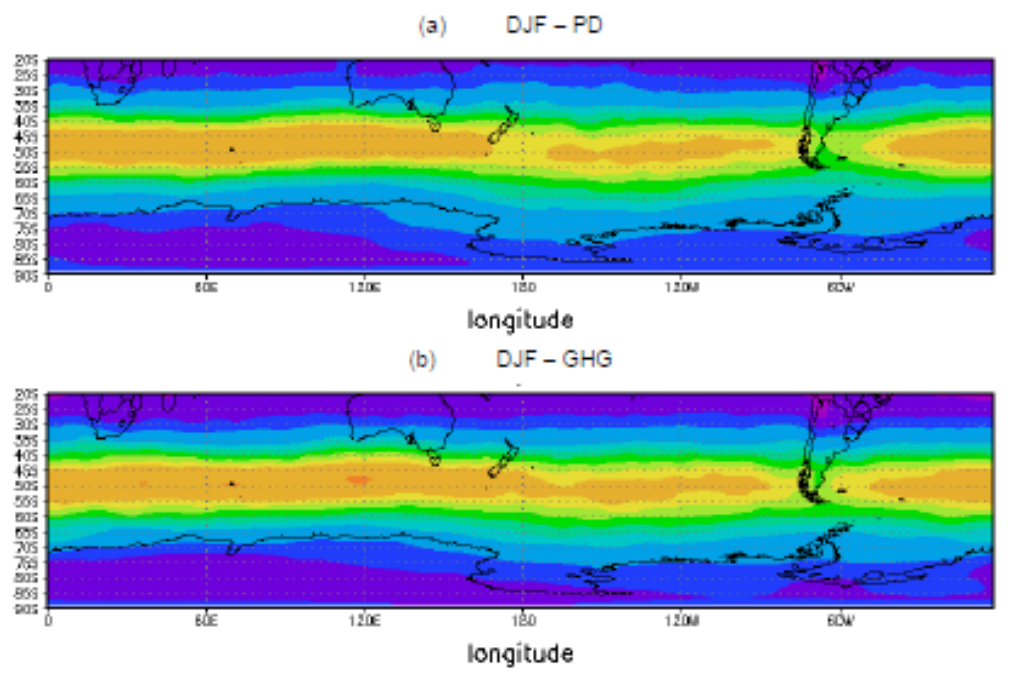

(c) JJA-PD

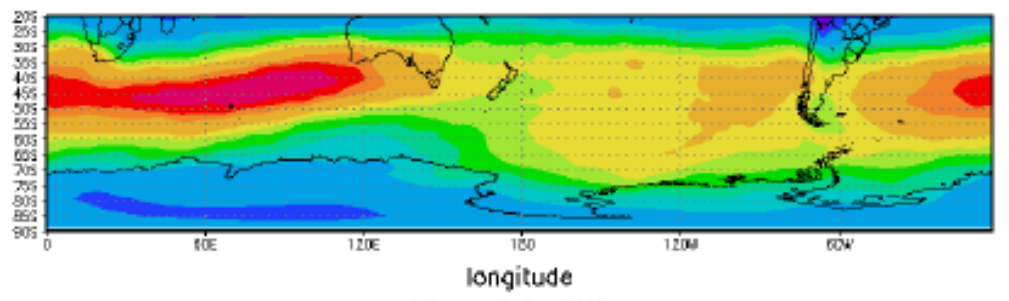

(d) JJA-GHG

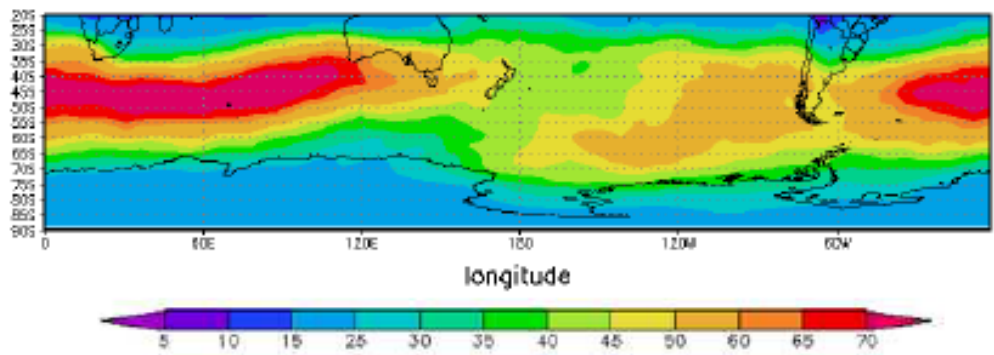

Fonte: Dos autores. 
A Figura 3 mostra o Fluxo de E em 500 hPa para simulações PD e GHG nos meses de verão e inverno no Hemisfério Sul. Nas Figuras são mostrados os valores do vento zonal médio, u (região colorida ao fundo da ilustração) e a convergência/divergência em vetores (o tamanho dos vetores representa a intensidade dos mesmos).

Para os meses de verão (Figura 3 a, b), observa-se as maiores divergências em 50 ${ }^{\circ} \mathrm{S}$ desde o sul do Oceano Índico até o sul da Austrália. Para a simulação GHG nota-se o perfil semelhante porém alongando-se até a Nova Zelândia. A intensidade e a divergência do fluxo não parecem aumentar consideravelmente para um cenário futuro. O padrão espacial parece semelhante, no entanto identifica-se uma ampliação na sua extensão longitudinalmente em relação à simulação PD.

Nas Figuras 3 (c) e (d), observa-se para os meses de inverno, uma atuação expressivamente mais intensa entre as latitudes de $30^{\circ} \mathrm{S}$ e $50^{\circ} \mathrm{S}$, sobretudo no Oceano Índico e oeste da Austrália, onde a divergência e a intensidade são bastante pronunciadas. No cenário de GHC esse padrão é mais robusto, com maiores valores de intensidade e divergência e mudança da sua distribuição para latitudes de $35^{\circ} \mathrm{S}$ e $55^{\circ} \mathrm{S}$. Para as duas simulações o Oceano Atlântico também apresenta uma região de divergência, embora sua intensidade seja mais intensa.

Sabendo-se que E mede a propagação dos vórtices alongados meridionalmente em relação ao fluxo de Oeste médio, nota-se na Figura 3 que as regiões de maior divergência do fluxo há um aumento da força horizontal da circulação média reforçando a tendência dos turbilhões em aumentar a oeste do fluxo médio.

As análises podem ser confirmadas para um cenário de aquecimento global através das anomalias (diferença entre GHC e PD) apresentadas na Figura 4. Observa-se que a maior divergência encontra-se sobre o Oceano Índico indicando que os vórtices estão contribuindo para acelerar o fluxo de Oeste na faixa de atuação dos ST em ambas as estações. Isso ocorre principalmente no Oceano Índico, sul da Austrália e Nova Zelândia, no inverno, onde os ventos intensificam-se até aproximadamente $5 \mathrm{~m}^{2} \mathrm{~s}^{-2} \mathrm{em}$ um cenário GHG (Figura 4). 
Figura 3 - Vetor $\vec{E}\left(\mathrm{~m}^{2} \mathrm{~s}^{-2}\right) 500 \mathrm{hPa}$. (a) Dados de reanálise ERA-40 em DJF; (b) Dados simulados pelo modelo ECHAM5/MPI-ON para GHG em DJF; e (c) e (d) O mesmo para os meses de inverno.

(a) DJF-PD

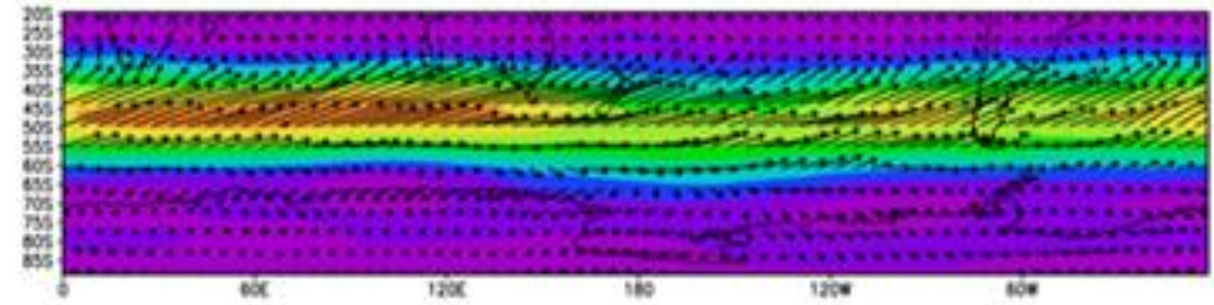

(b) DUF - GHG

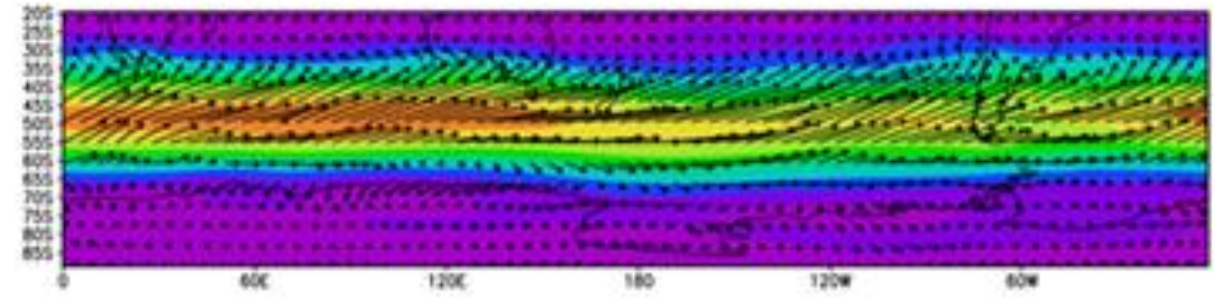

(c) JIA - PD

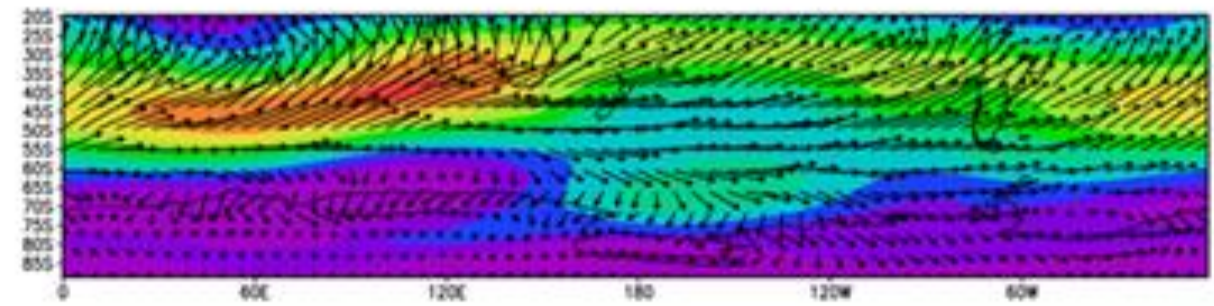

(d) GHG - JHA

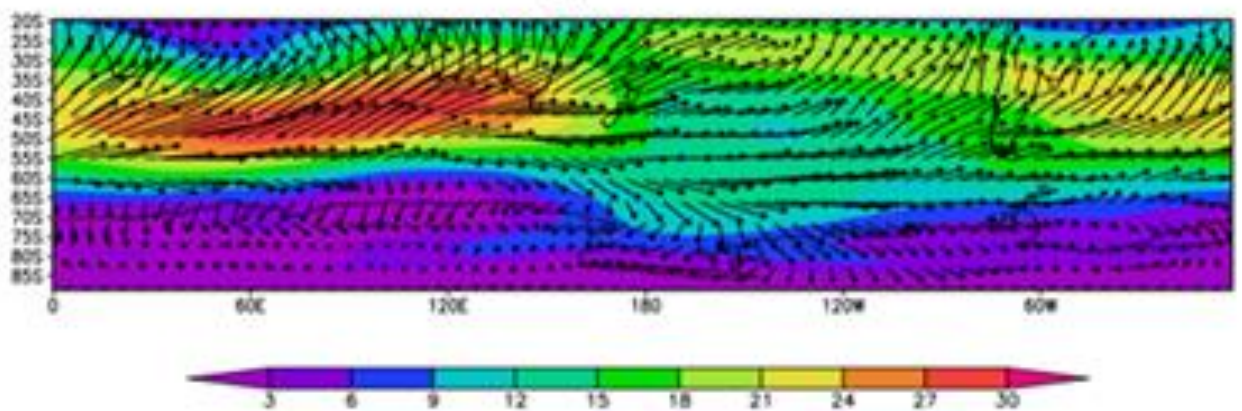

(a) Dados de reanálise ERA-40 em DJF; (b) Dados simulados pelo modelo ECHAM5/MPI-ON para GHG em DJF; e (c) e (d) O mesmo para os meses de inverno.

Fonte: Dos autores. 
Figura 4 - Anomalias Vetor $\vec{E}\left(\mathrm{~m}^{2} \mathrm{~s}^{-2}\right) 500 \mathrm{hPa}$. (a) DJF; (b) JJA.

(a) DJF

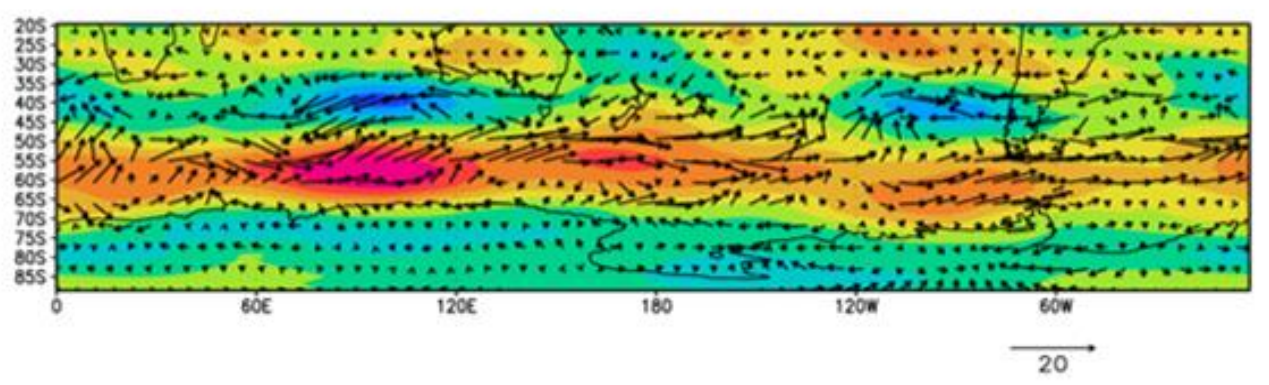

(b) JJA

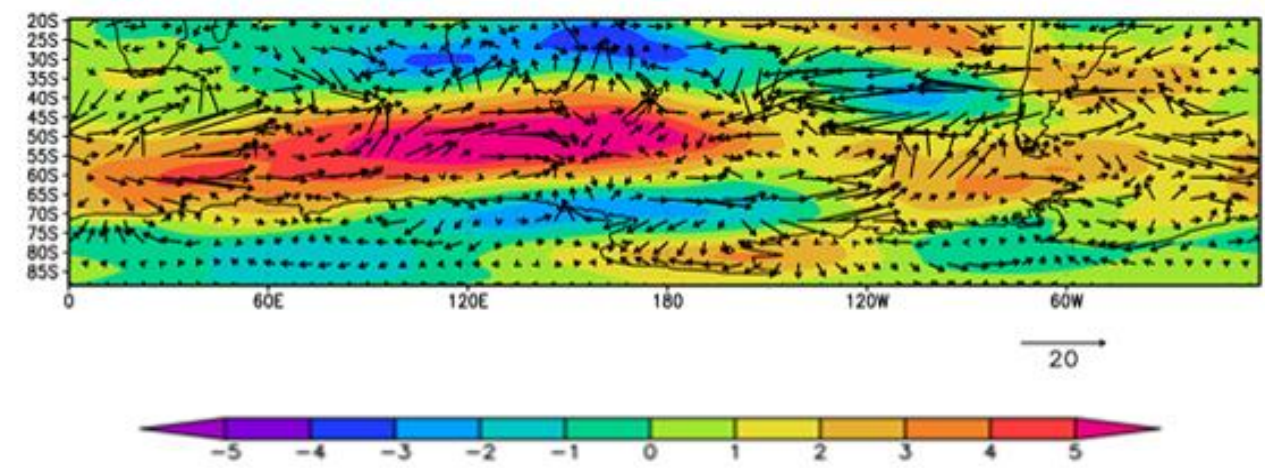

Fonte: Dos autores.

\section{CONCLUSÕES}

A energia cinética associada ao deslocamento dos ST mostra um aumento da atividade sobre o Pacifico Central, bem como uma intensificação do fluxo de Oeste. Assim, em um cenário futuro, o fluxo médio interage com os ST, principalmente nos meses de inverno. Esta interação entre os fluxos médios e transientes pode auxiliar no deslocamento mais para o sul em um cenário de aquecimento global.

Os padrões dominantes de EKE mostram que, para um cenário futuro, as maiores tempestades estariam distribuídas no verão a partir de $60^{\circ} \mathrm{E}$ até $120^{\circ} \mathrm{W}$, sugerindo maior impacto sobre o sul da Austrália e Nova Zelândia. Nos meses de inverno as maiores tempestades apresentam-se entre o sul da África, Oceano Índico e sul da Austrália e bem mais evidentes no Oceano Atlântico. 
A partir de análises do vetor $\vec{E}$ associadas com demais análises conclui-se então, que os ST estão intimamente ligados ao fluxo de oeste, podendo-se afirmar que em um cenário futuro haverá uma intensificação desse transporte e os mesmos deslocar-se-ão mais a sul. Dessa forma, espera-se que haja implicações nos abastecimentos de água de algumas regiões como o Sul da África onde maior parte da população é dependente da precipitação da região.

Na região Sul do Brasil, os resultados indicam aumentos nas chuvas durante os meses de verão, contrastando com uma diminuição na frequência e aumento na intensidade no inverno. Sugerimos que essas mudanças podem impactar a dinâmica climática do litoral sul brasileiro, com magnitude ainda desconhecida.

\section{REFERÊNCIAS}

BENGTSSON, L. et al. Storm Tracks and Climate Change. Journal of Climate, 19(15): 3518-3543, 2006.

FREITAS, Rose Ane Pereira et al. The Storm Tracks response to changes in atmospheric greenhouse gas concentration at the south of Brazil and Southwest Atlantic Ocean. Atmospheric and Climate Sciences, v. 9, n. 4, p. 545-557, 2019. DOI: $10.4236 /$ acs.2019.94035

HALL, N.M.J. et al. Storm Tracks in a high-resolution GCM with doubled carbon dioxide. Quarterly Journal of the Royal Meteorological Society. v. 128, p. 12091230, 1994.

HARTMANN, D.L. Global physical climatology. International Geophysics Series, v. $56,1994$.

HOSKINS, Brian J.; VALDES, Paul J. On the existence of storm-tracks. Journal of Atmospheric Sciences, v. 47, n. 15, p. 1854-1864, 1990.

IPCC, 2021: Climate Change 2021: The Physical Science Basis. Contribution of Working Group I to the Sixth Assessment Report of the Intergovernmental Panel on Climate Change [Masson-Delmotte, V., P. Zhai, A. Pirani, S.L. Connors, C. Péan, S. Berger, N. Caud, Y. Chen, L. Goldfarb, M.I. Gomis, M. Huang, K. Leitzell, E. Lonnoy, J.B.R. Matthews, T.K. Maycock, T. Waterfield, O. Yelekçi, R. Yu, and B. Zhou (eds.)]. Cambridge University Press. In Press.

JUNGCLAUS, J.H, et al. Ocean circulation and tropical variability in the coupled model ECHAM5/MPI-OM, Journal of Climate, v. 19, pp. 3952-3972, 2006. 
JUNGCLAUS, J.H. et al. Ocean circulation and tropical variability in the coupled model ECHAM5/MPI-OM. Journal of Climate, v. 19, p. 3952-3972, 2006.

KUTZBACH, J.E. Empirical eigenvectores of sea level pressure, surface temperature andprecipitation complexes over North America. Journal of Applied Meteorology. 6:791-802 Oct. 1967.

KUTZBACH, J.E. Empirical eigenvectores of sea level pressure, surface temperature andprecipitation complexes over North America. Journal of Applied

Meteorology. v. 6, p. 791-802, 1967.

LAU, N.C. Variability of the observed mid latitude Storm Tracks in relation to lowfrequency changes in the circulation pattern. Journal of the Atmospheric Sciences, v. 45, p. 2718-2743, 1988.

LEGLER, D.M. Empirical orthogonal function analysis of wind vectors over the tropical Pacific region. Bulletin of the American Meteorological Society, v. 64, $\mathrm{n}$. 3, p. 243-241, 1983.

LEHMANN, Jascha et al. Future changes in extratropical Storm Tracks and baroclinicity under climate change. Environmental Research Letters, v. 9, n. 8, p. 084002, 2014.

MACHADO, Jeferson Prietsch; JUSTINO, Flávio; SOUZA, Caio Demétrio. Influence of El Niño-Southern Oscillation on baroclinic instability and Storm Tracks in the Southern Hemisphere. International Journal of Climatology, v. 41, p. E93-E109, 2021.

MCBEAN, Gordon. Climate change and extreme weather: a basis for action. Natural Hazards, v. 31, n. 1, p. 177-190, 2004.

MESQUITA, M.S. Tracking summer extra-tropical storms: a climatological overview and variability in the Northern Hemisphere. University of Bergen, Norway. 2006.

O'GORMAN, Paul A. Understanding the varied response of the extratropical Storm Tracks to climate change. Proceedings of the National Academy of Sciences, v. 107, n. 45, p. 19176-19180, 2010.

PEZZA, A.B.; SIMMONDS, I. The first south atlantic hurricane unprecedented blocking, iow shear and climate change. Geophysical Research Letters, L15712, p. 1-5, 2005.

PEZZI, L.P.; SOUZA, E.B. O uso da temperatura da superfície do mar em estudos climáticos. In: SOUZA, R.B. (Org.). Oceanografia por satélites. São Paulo: Oficina de Textos. P. 117-133. 2005.

ROBERTS, Malcolm John et al. Projected future changes in tropical cyclones using the CMIP6 HighResMIP multimodel ensemble. Geophysical research letters, v. 47, n. 14, p. e2020GL088662, 2020. 
STERL, A. et al. Simulações parte do Projeto ESSENCE. Instituto Max-Planck de Meteorologia. Disponível em: <www.knmi.nl/ sterl/Essence>. Acesso em: 05 jun. 2010.

TINGS, M.F.; HELD, I.M. The stationary wave response to a tropical SST anomaly in an idealized GCM. Journal of the Atmospheric Sciences, v. 47, p. 2546-256.

UPPALA, Sakari M. et al. The ERA-40 re-analysis. Quarterly Journal of the Royal Meteorological Society: A journal of the atmospheric sciences, applied meteorology and physical oceanography, v. 131, n. 612, p. 2961-3012, 2005.

Recebido em: 10/10/2021

Aprovado em: 12/11/2021

Publicado em: 18/11/2021 